\title{
Electrode Modification by Long-Chain, Dialkyl Disulfide Reagent Having Terminal Dinitrophenyl Group and Its Application to Impedimetric Immunosensors
}

\author{
Hiroaki Taira*, Koji Nakano**, Mizuo Maeda* and Makoto TaKagi* \\ *Department of Chemical Science and Technology, Kyushu University, \\ Hakozaki, Fukuoka 812, Japan \\ **Laboratory of Chemistry, College of General Education, Kyushu University, \\ Ropponmatsu, Fukuoka 812, Japan
}

\begin{abstract}
A long-chain, dialkyl disulfide having a terminal dinitrophenyl (DNP) group was newly synthesized for modification of a surface of gold with chemisorbed, self-assembled organic monolayers. The resulting monolayer was characterized by infrared spectroscopy of reflection absorption spectroscopy mode, by cyclic voltammetric measurement and by electrical impedance measurement. Coupled with impedance measurements, a possible application of the modified electrode system to impedimetric immunosensors was demonstrated. The sensor gave electrical response to anti-DNP antibody at $10-10^{3} \mathrm{ng} \mathrm{cm}^{-3}$ concentration.
\end{abstract}

Keywords Bifunctional alkanethiol, chemisorption, self-assembled monolayer, modified gold electrode, impedimetric immunosensor

Recent advances in chemically modified electrodes (CMEs) have stimulated considerable research interest in their application to electrochemical sensors. ${ }^{1}$ Central to both the fundamental study of CMEs and their sensor applications is the rational manipulation of the electrode surface functionality by immobilizing selected types of molecules. One of the recent works in this area has focused on the monolayer formation on surfaces of gold from organosulfur precursors. ${ }^{2}$

Stable attachment of organic molecules to gold and other metal surfaces is attained through the use of reagents having thiol, sulfide, disulfide, or other sulfurcontaining head groups by immersing gold substrate into their dilute solutions. In case of compounds having a long-chain alkyl group, monolayers with highly-ordered, well-defined structure are formed spontaneously. This attachment has been described as due to the formation of some type of thiolate complex on the gold surface, and therefore, the use of sulfur-containing species as anchors on gold seems to be especially attractive for CMEs' application because of the stability of the surface complex formed.

However, formation of densely-packed hydrocarbon monolayers becomes a disadvantage to electrochemical application. The redox reaction of the target analyte is blocked almost completely on the modified electrode. Use of functionalized thiols with redox-active groups ${ }^{3}$ can be one promising way to overcome this difficulty, because such modifiers can mediate an electron transfer between the gold substrate and the analyte in solution, making the amperometric measurement feasible. There can be another sensing approach which now makes use of the electrically insulating property itself of the modified gold surface. It is an impedimetric sensing ${ }^{4}$, where the change in electrical impedance characteristics (change in dielectric property) of the modified surface layer is used to sense the chemisorbed species on the sensing electrode surface. In the present study, we synthesized a dialkyl disulfide compound having terminal dinitrophenyl groups (dinitrophenyl: DNP) (1) and studied some fundamental physico-chemical characteristics of the 1modified gold electrode for its possible application to impedimetric sensing of protein (anti-DNP antibody).

Antigen and antibody measurements are important in clinical chemistry, physiology, and modern biotechnology. However, if one considers their determination by electrochemical technique, a problem arises because antibody-antigen reactions are usually not directly linked to redox processes. In addition, the reactions are stoichiometric and do not involve chemical amplification. This is in a marked contrast to enzyme reactions which often give potentiometrically or amperometrically measurable reaction products in an amplified manner. Reflecting these situations, considerable efforts have been made to date to develop electrochemical immunosensing devices such as electrochemical enzyme immunoassay $^{5}$, potentiometric ionophore-modulation immunoassay $^{6}$, and piezoelectric systems. ${ }^{7}$

On the other hand, a different approach has recently been made to detect the antibody-antigen reactions 
electrochemically. An immunochemical reaction is carried out on an electrode surface and the change in dielectric property of the surface layer is measured. The effort is to exploit capacitative or impedimetric immunosensors. Martelet $e t$ al. reported quite simple, direct detection of immunospecies by capacitance measurements on a silicon/silicon dioxide/immobilized antibody/electrolyte heterostructure. ${ }^{8}$ They considered the electrode surface as having an ideally blocked layer structure, each layer carrying a specific electric capacitance in a series of combinations. In order for this type sensory technique to have generality, it seems important that the quality of such surface layer properties as structural homogeneity and integrity are controlled reproducibly in the electrode fabrication process. Further, it is emphasized that one may be able to evaluate a.c. parameters of the fabricated layer properly only when the nature of the electrical equivalent circuit is reasonably understood on the chemical basis on the electrode. ${ }^{4}$

We supposed that such factors in electrode fabrication as pointed out above are most conveniently studied on a
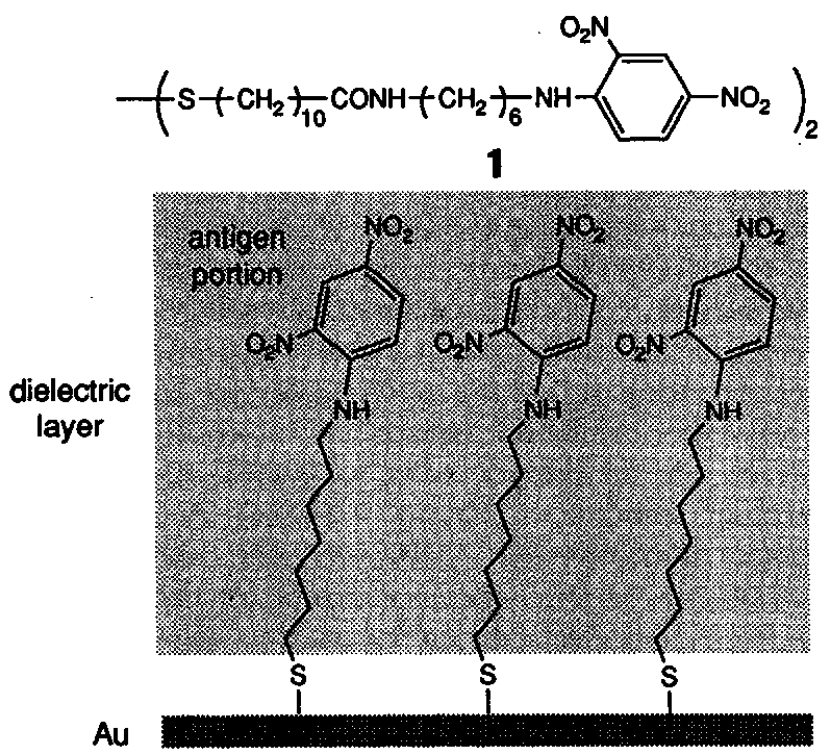

Fig. 1 Structure of 1 and schematic illustration of chemisorbed 1-monolayer on gold surface. gold electrode by thiol modification technique. In the present study, we synthesized a dialkyl disulfide compound carrying a terminal dinitrophenyl group (1). An organic monolayer therefrom was developed on the surface of the gold substrate and was characterized by Fourier-transform infrared spectroscopy (FT-IR) and electrochemical methods. A possible application to an electrochemical immunosensor based on an impedimetric principle was also investigated.

\section{Experimental}

\section{Chemical and immunochemical reagents}

Chemicals for synthetic use were of extra pure grade. All other chemicals were guaranteed reagents and were used without further purification. Immunochemical reagents, monoclonal anti-DNP antibody (Sigma, from mouse sources: subclass, IgE) and monoclonal anti-IgE antibody (from rat sources, Cosmo Bio Co.) were used as received.

Reagent 1 was prepared by a synthetic reaction in Scheme 1.

\section{$N$-(6-Aminohexyl)-2,4-dinitroaniline}

1-Chloro-2,4-dinitrobenzene was reacted with a slight molar excess of 1,6-diaminohexane in dichloromethane for $24 \mathrm{~h}$ at room temperature. ${ }^{9}$ After evaporation of the solvent and the excess 1,6-diaminohexane under reduced pressure, the residue was subjected to silica gel column chromatography to obtain $N$-(6-aminohexyl)-2,4dinitroaniline.

\section{1,11'-Dithiodiundecanoic acid}

11-Bromoundecanoic acid was allowed to react with an equimolar amount of thiourea in ethanol to give a thiouronium salt, which was then hydrolyzed with aqueous potassium hydroxide to give 11-mercaptoundecanoic acid. ${ }^{10}$ The thiol obtained was then oxidized with hydrogen peroxide ${ }^{11}$ to give the disulfide.

\section{Bis(10-(N-(6-(2,4-dinitrophenylamino)hexyl)carbamoyl) decyl)disulfide(1)}

While cooling externally in an ice-bath, $11,11^{\prime}$-dithiodiundecanoic acid $(0.15 \mathrm{~g})$ was treated with $N$-(6-aminohexyl)-2,4-dinitroaniline $(0.2 \mathrm{~g})$ in $20 \mathrm{~cm}^{3}$ dichloromethane in the presence of $N, N^{\prime}$-dicyclohexylcarbodiimide

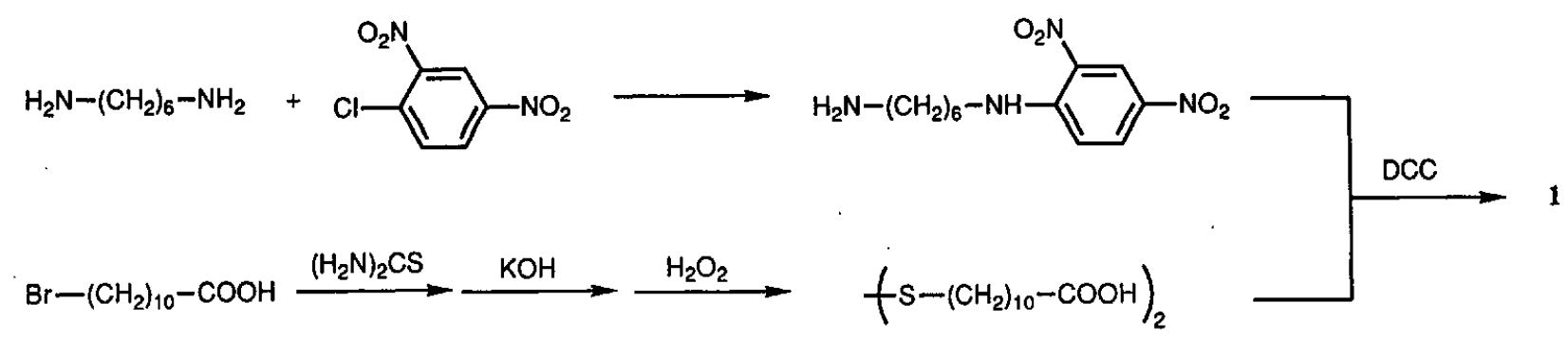


$(0.15 \mathrm{~g})$ for $24 \mathrm{~h}$. After evaporation of the solvent, the residue was subjected to column chromatography (silica gel) to obtain 1: yellow powder; $\mathrm{mp} 89.5-90^{\circ} \mathrm{C}$. ${ }^{1} \mathrm{H}-$ nmr (HITACHI, Ltd., R-24B) (60 MHz, $\mathrm{CDCl}_{3} ; \delta$ ppm downfield of tetramethylsilane) $1.42\left(\mathrm{~m}, 28 \mathrm{H}, \mathrm{CH}_{2}\right), 2.17$ (m, 3H, $\left.\mathrm{CH}_{2}-\mathrm{CO}\right), 2.64\left(\mathrm{t}, J=7 \mathrm{~Hz}, 2 \mathrm{H}, \mathrm{S}-\mathrm{CH}_{2}\right), 3.32(\mathrm{~m}$, $\left.4 \mathrm{H}, \mathrm{NH}-\mathrm{CH}_{2}\right), 6.05(\mathrm{t}, J=6 \mathrm{~Hz}, 1 \mathrm{H}, \mathrm{CO}-\mathrm{NH}), 6.91$ (d, $J=9.6 \mathrm{~Hz}, 1 \mathrm{H}, \mathrm{C}-6 \mathrm{CH}), 8.19$ (dd, $J=2.8,9.6 \mathrm{~Hz}, 1 \mathrm{H}$, $\mathrm{C}-3 \mathrm{CH}$ ), 8.51 (t, $\left.J=5 \mathrm{~Hz}, 1 \mathrm{H}, \mathrm{NH}-\mathrm{C}_{6} \mathrm{H}_{3}\left(\mathrm{NO}_{2}\right)_{2}\right), 9.00$ (d, $J=2.8 \mathrm{~Hz}, 1 \mathrm{H}, \mathrm{C}-3, \mathrm{CH})$. IR (KBr), 2925, 2851, 1630, $1588,1542,1527,1422$, and $1352 \mathrm{~cm}^{-1}$. Elemental analysis: calculated for $\mathrm{C}_{46} \mathrm{H}_{74} \mathrm{O}_{10} \mathrm{~N}_{8} \mathrm{~S}_{2}$ (found); $C, 57.35 \%$ (56.91\%); H, 7.74 (7.74); N, 11.64 (11.64).

\section{FT-IR measurement}

IR spectral measurements, both transmission mode in $\mathrm{KBr}$ pellet and RAS (reflection absorption spectroscopy) mode, were performed on a Nicolet $510 \mathrm{M}$ spectrometer equipped with a MCT detector. The transmittance spectrum was measured at a spectral resolution of $4 \mathrm{~cm}^{-1}$ with 10 scans. For RAS mode, gold substrates were first prepared by vacuum evaporation onto slide glasses. A monolayer of 1 was prepared by immersing a freshly prepared substrate into a 1,4-dioxane solution of 1 (concentration, $10 \mathrm{mM}$ ) for $24 \mathrm{~h}$ at room temperature. The substrate was carefully rinsed with 1,4-dioxane, dried under vacuum and was used for measurements immediately. The $p$-polarized radiation was introduced on the sample at $85^{\circ}$ off the surface normal and data were collected at a spectral resolution of $4 \mathrm{~cm}^{-1}$ with 1095 scans.

\section{Electrochemical measurements}

A gold disk electrode (diameter $1.6 \mathrm{~mm}$, Bioanalytical Systems Co.) was used as working electrode through out the present electrochemical measurements. The electrode surface was polished.to a mirror finish with alumina powders of successively finer grades (Buehler Co.) and was chemically modified with the monolayer of 1 by the same procedure as described above.

Cyclic voltammetric measurements were performed by using a Solartron Co. Model 1286 potentiostat with a design of a three-electrode system. A Pt plate $(10 \times 10$ $\mathrm{mm}$ ) and a standard $\mathrm{Ag} / \mathrm{AgCl}$ (saturated $\mathrm{KCl}$ ) electrodes were used as counter and reference electrodes, respectively.

The apparatus used for impedance measurements ${ }^{12}$ was composed of a Solartron Co. Model 1250A frequency response analyzer coupled with a Model 1286 potentiostat. A three-electrode configuration using a counter electrode ( $\mathrm{Pt}$ plate, $10 \times 10 \mathrm{~mm}$ ) and a standard $\mathrm{Ag} / \mathrm{AgCl}$ reference electrodes (saturated $\mathrm{KCl}$ ) was employed for impedance measurements.

Frequency response analysis (FRA) involves a study of the way in which the total impedance of the cell varies with frequency. The working electrode is held at a constant potential with respect to a reference electrode, and a superimposed overlapping small perturbation voltage is applied between the working electrode and the counter electrode. The electrical response of the circuit is then measured and analyzed. In practice, an opencircuit potential of the working electrode was first determined before each FRA measurement. The opencircuit potential was monitored against a reference electrode under nitrogen until it became constant (typically $30 \mathrm{~min}$ ). The potential of the working electrode was then held at this value, and a $50 \mathrm{mV}$ (rms) sinusoidal wave ranging from $0.1 \mathrm{~Hz}$ to $65 \mathrm{kHz}$ was applied. All experiments were made at a constant temperature of $25 \pm 0.2^{\circ} \mathrm{C}$.

\section{Results and Discussion}

\section{Synthesis of immuno-affinity anchoring reagent 1}

In designing the reagent 1 , we took the binding affinity between the antibody and a series of hapten molecules into consideration. Herman et al. ${ }^{13}$ investigated the affinities of anti-2,4-DNP antibodies to a variety of 2,4dinitrobenzene derivatives and found $\varepsilon-N-\mathrm{DNP}-\mathrm{L}-\mathrm{lysine}$ to have the highest affinity to the antibody. Because antigens typically have a molecular weight greater than 1500 to elicit efficient antibody formation, antibodies to small molecules, such as DNP moiety, are obtained by chemically attaching the molecule to some larger carrier antigen, usually a protein. Such chemical modifications of proteins, in general, are suggested to occur at an $\varepsilon$-amino group of lysine. This explains the high antibody affinity of $\varepsilon$-DNP-L-lysine. Based on this consideration, we designed the immuno-affinity anchoring reagent 1 which incorporated $N$-alkyl-2,4-dinitroaniline structure.

\section{Characterization of organic monolayer on gold by $I R$ spectroscopy}

Figure 2 shows IR spectra found for 1 in monolayer state on gold substrate and in microcrystalline state in a $\mathrm{KBr}$ disk. The RA spectrum of the 1-treated gold substrate shows fundamentally a spectrum similar to the transmittance spectrum of microcrystalline 1 . In the high-frequency region, RA spectrum shows a broad peak around $3300 \mathrm{~cm}^{-1}$ which can be assigned to overlapped $\mathrm{N}-\mathrm{H}$ stretching modes of the amino and amide groups. In the transmittance spectrum in $\mathrm{KBr}$ disk, this band is further obscured by a broad $\mathrm{O}-\mathrm{H}$ stretching band due to the adsorbed moisture. Two peaks at $2933 \mathrm{~cm}^{-1}$ and $2860 \mathrm{~cm}^{-1}$ can be identified as $\mathrm{C}-\mathrm{H}$ stretches for antisymmetrical and symmetrical modes of the connecting methylene group, respectively. In the mid-frequency region, which contains most of the information regarding the structural feature of 1 , an intense peak at $1629 \mathrm{~cm}^{-1}$ can be assigned to $\mathrm{C}=\mathrm{O}$ stretch mode of amide group. Two peaks at 1593 and $1344 \mathrm{~cm}^{-1}$ are $\mathrm{NO}_{2}$ stretches of antisymmetrical and symmetrical modes, respectively. A broad band at $1427 \mathrm{~cm}^{-1}$ can be identified as $\mathrm{CH}_{2}$ scissoring mode.

The positions of peak frequencies of $\nu\left(\mathrm{CH}_{2}\right)$ modes give insight into the "crystalline-like"or "liquid-like" nature of the monolayer. ${ }^{15}$ From the literature ${ }^{14}$, the 

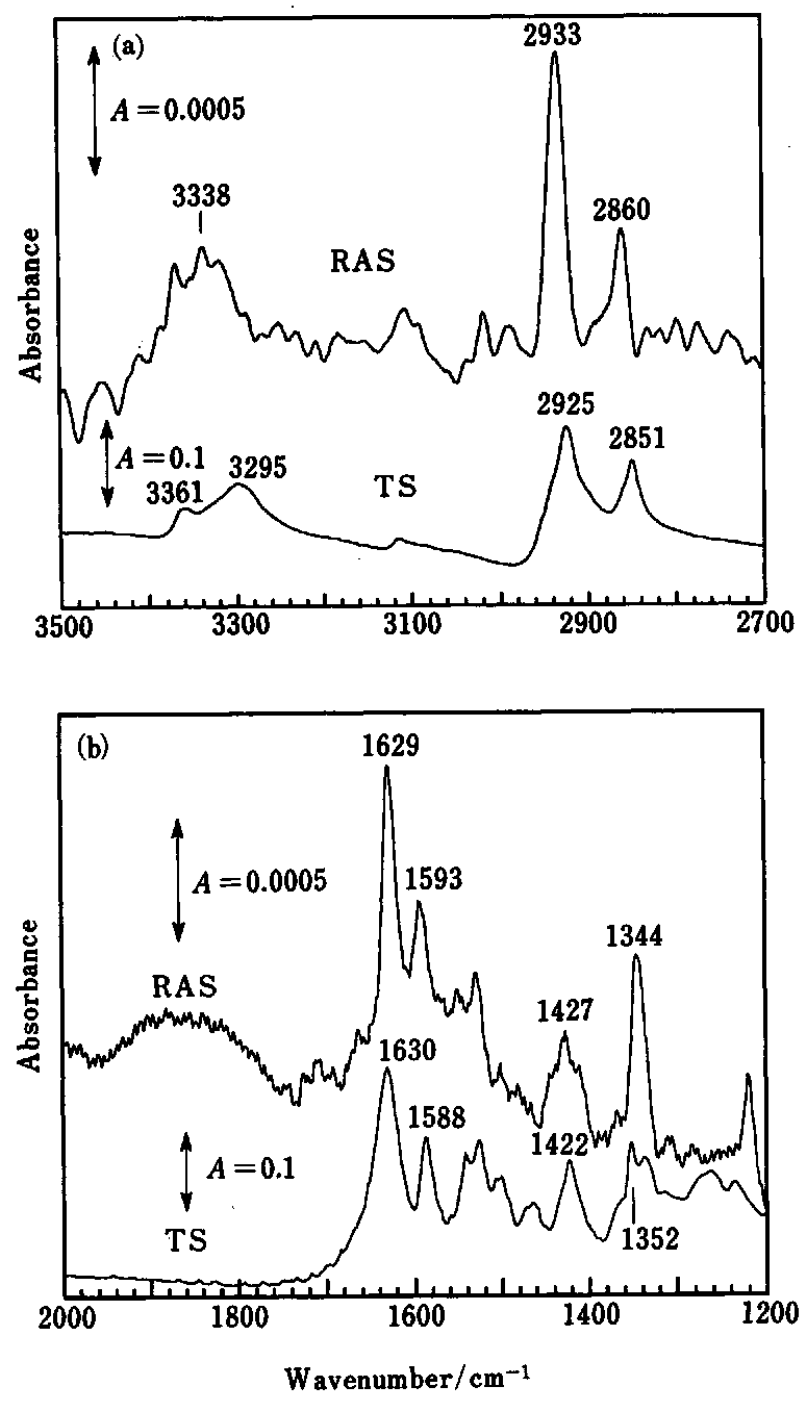

Fig. 2 FT-IR spectra in (a) high-frequency region and in (b) mid-frequency region. RAS denotes reflection absorption spectra of 1-treated gold substrate and TS denotes transmittance spectra of microcrystals of 1 dispersed in a $\mathrm{KBr}$ disk.

peak position of the $\nu_{\text {as }}\left(\mathrm{CH}_{2}\right)$ mode of crystalline polymethylene chain $\left(2920 \mathrm{~cm}^{-1}\right)$ is $8 \mathrm{~cm}^{-1}$ lower than that for the liquid state $\left(2928 \mathrm{~cm}^{-1}\right)$, whereas for $\nu_{\mathrm{s}}\left(\mathrm{CH}_{2}\right)$ mode the peak position is $6 \mathrm{~cm}^{-1}$ lower in the crystalline state $\left(2850 \mathrm{~cm}^{-1}\right)$ than that in the liquid $\left(2856 \mathrm{~cm}^{-1}\right)$. Compound 1 contains two different methylene chains. Thus, some ambiguities are introduced into the interpretation of the $\mathrm{CH}_{2}$-stretches. However, the peak positions of $\nu_{\text {as }}\left(\mathrm{CH}_{2}\right)$ mode and $\nu_{\mathrm{s}}\left(\mathrm{CH}_{2}\right)$ mode for the monolayer 1 are $8 \mathrm{~cm}^{-1}$ and $9 \mathrm{~cm}^{-1}$ higher than those for microcrystalline 1 , respectively. These data suggest that 1 forms "liquidlike" monolayers on gold surface, in contrast to those monolayers from simple alkanethiols with long alkyl chain. ${ }^{15}$ Based on Corey-Pauling-Koltun (CPK) model building, the cross section of the DNP group was estimated to be $38 \AA^{2}$ (8 $\AA \times 4.8 \AA$ ), while it was only $14 \AA^{2}$ $(3.8 \AA \times 3.6 \AA)$ for polymethylene chain. This incompatibility in molecular dimension along the terminally dinitrophenyl-substituted alkyl chain should lead to the "liquid-like" nature of the polymethylene chain in the monolayer formed from 1 , since the placement of a bulky DNP group upon the alkyl chain layer inevitably precludes the closest packing of polymethylene chains.

Anyhow, the results obtained above strongly suggest that 1 also adsorbs onto gold surfaces in a similar manner to simple alkanethiols. Consequently, we are now able to modify gold surfaces with an organic monolayer film having dinitrophenyl terminal groups.

\section{Characterization of monolayer by cyclic voltammetry}

In order to probe the adsorption behavior of 1 and the nature of the resulting monolayer, we studied 1-modified gold electrode by cyclic voltammetric and impedance measurements. The former technique gave information on the extent of monolayer coverage on the electrode surface, while the latter gave insight into physicochemical nature of the monolayer.

Figure 3 shows cyclic voltammograms measured on 1modified gold electrode in $0.1 \mathrm{M} \mathrm{H}_{2} \mathrm{SO}_{4}$ solution. Under such strong acidic conditions, the monolayers were expected to deteriorate rapidly, but the cyclic voltammograms were not affected on the repeated potential scanning. The gold oxide removal peak at around $1.0 \mathrm{~V}$ was integrated by a graphical procedure, and the integrated value was compared with the corresponding value from the untreated bare gold electrode to estimate the surface coverage ratio, $\theta(\theta=0$ for bare electrode; $\theta=1$ for completely covered electrode). ${ }^{16}$ To confirm the reproducibility of surface modification and $\theta$ measurement, the same gold electrode was repolished, surface-modified (treatment with 1), and subjected to the same voltammetric study. Such repeated $\theta$ measurements gave $\theta$ values of 0.14 , $0.19,0.31$ for three independent runs. For comparison, dodecanethiol was also used as electrode modifier and a

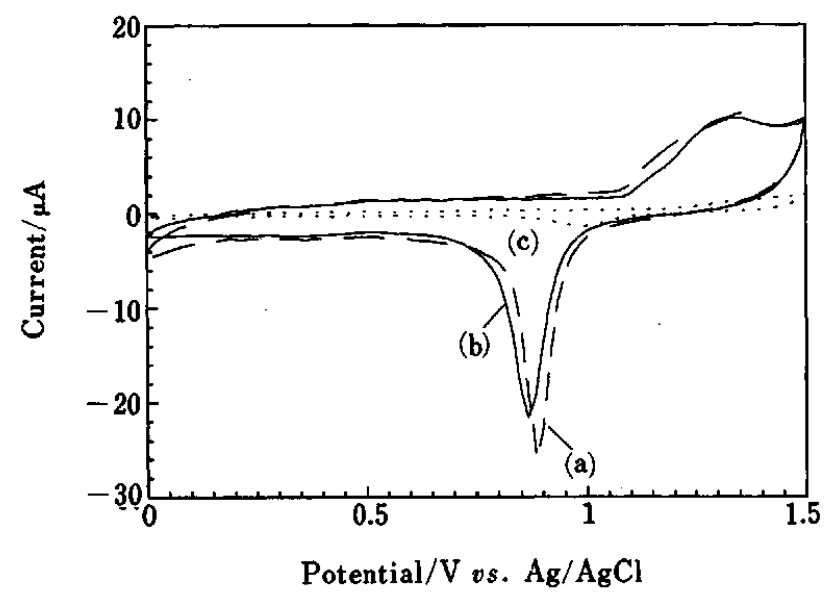

Fig. 3 Cyclic voltammograms for (a) bare gold electrode, (b) the gold electrode after treatment with 1 (the voltammogram of its unmodified state is (a)) and (c) $\mathrm{C}_{12} \mathrm{H}_{25} \mathrm{SH}$-modified electrode (the voltammogram of its unmodified state is not shown). Electrolyte solution, aqueous $0.1 \mathrm{M} \mathrm{H}_{2} \mathrm{SO}_{4}$; scan rate, $100 \mathrm{mV} \mathrm{s}^{-1}$; temperature, $25^{\circ} \mathrm{C}$. 
$\theta$ value of 0.85 was obtained.

Surface coverage on gold substrate should suffer such experimental uncertainties as the roughness of (polished) gold surface, the extent of activation of $\mathrm{Au}$ surface (cleanness of surface), and so on. Taking these factors into consideration, the reproducibility of surface coverage indicated above seems reasonably good. On the other hand, the low $\theta$ value for 1 relative to that of dodecanethiol monolayer may be ascribed to the steric hindrance due to the bulky DNP group. As already noted before, the cross section of the DNP group is estimated to be $38 \AA^{2}$ while it is only $14 \AA^{2}$ for polymethylene chain. This means that the effective (greatest) molecular cross section of 1 is 2.7 times larger than that of dodecanethiol. If one makes a simple assumption that $\theta$ value is reciprocally proportional to the greatest cross section within the molecular chain, the $\theta$ value expected for monolayer of 1 would be $1 / 2.7$ times that observed $(\theta=0.85)$ for dodecanethiol monolayer, that is, $0.85 \times(1 / 2.7)=0.32$. The experimental value agrees fairly well with this estimation.

Electron transfer measurements were then made in aqueous solution by using cyclic voltammetric techniques. A $\left[\mathrm{Fe}(\mathrm{CN})_{6}\right]^{3-/ 4-}$ redox couple was used since it represents an electrochemically reversible, welldefined, one-electron redox reaction with moderate normal potential $\left(E^{\prime}=0.21 \mathrm{~V}\right)$. Figure 4 shows typical cyclic voltammograms obtained with a bare gold electrode and a 1-modified gold electrode having a $\theta$ value of 0.31 . Although a small current due to ferrocyanide oxidation appears with a large overpotential, most of the current observed at the modified electrode is capacitative. Therefore, the monolayer of 1 on the gold electrode effectively blocks the electron transfer process to and from the solution species in spite of its low surface coverage ratio. This blocking effect may be partially due to the hydrophobic character of the monolayer.

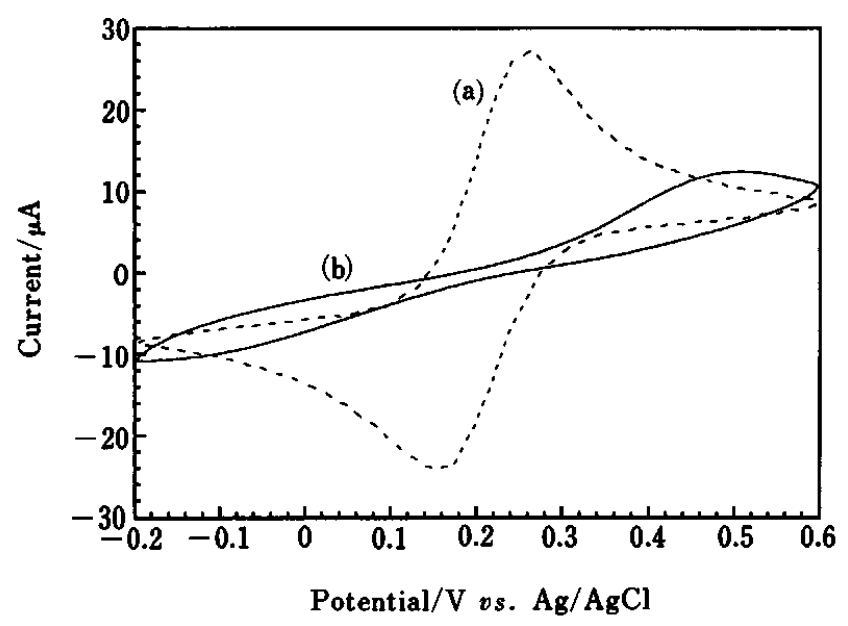

Fig. 4 Cyclic voltammograms for (a) bare Au electrode and (b) 1-modified gold electrode. Electrolyte solution: aqueous $0.1 \mathrm{M} \mathrm{KCl}$ containing $5 \mathrm{mM}$ each of $\mathrm{K}_{4}\left[\mathrm{Fe}(\mathrm{CN})_{6}\right]$ and $\mathrm{K}_{3}\left[\mathrm{Fe}(\mathrm{CN})_{6}\right]$. Scan rate, $50 \mathrm{mV} \mathrm{s}^{-1}$; temperature, $25^{\circ} \mathrm{C}$.
Characterization of monolayer by impedance spectroscopy

In the presence of redox-active species in solution, an impedance response of the electrode-solution system is conveniently expressed in terms of Randles' equivalent circuit. The circuit is illustrated below:

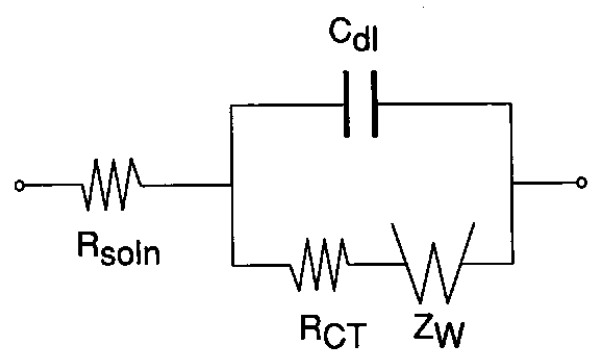

where $R_{\text {soln }}, C_{\mathrm{dl}}, R_{\mathrm{CT}}$ and $Z_{\mathrm{w}}$ represent a resistance of the electrolyte solution, a capacitance of the electrical double layer on the electrode surface, a resistance to redox (charge transfer) reaction at the electrode surface and a Warburg impedance, respectively. The Warburg impedance represents a resistance when redox-active species diffuse through the boundary layer on the electrode surface. In Randles' equivalent circuit, Faradaic impedance (an electrical impedance which accompanies Faradaic current) is represented by a serial combination of $R_{\mathrm{CT}}$ and $Z_{\mathrm{w}}$.

Figure 5 shows complex impedance diagrams obtained in our system for bare and modified electrodes in the presence of $\left[\mathrm{Fe}(\mathrm{CN})_{6}\right]^{3-14-}$ redox couple. For ordinary redox processes on metal electrodes, Randles' equivalent circuit gives a complex impedance diagram which shows the so-called kinetic semi-circle in a high frequency region, since the diffusion of redox species does not contribute much to the total impedance and the

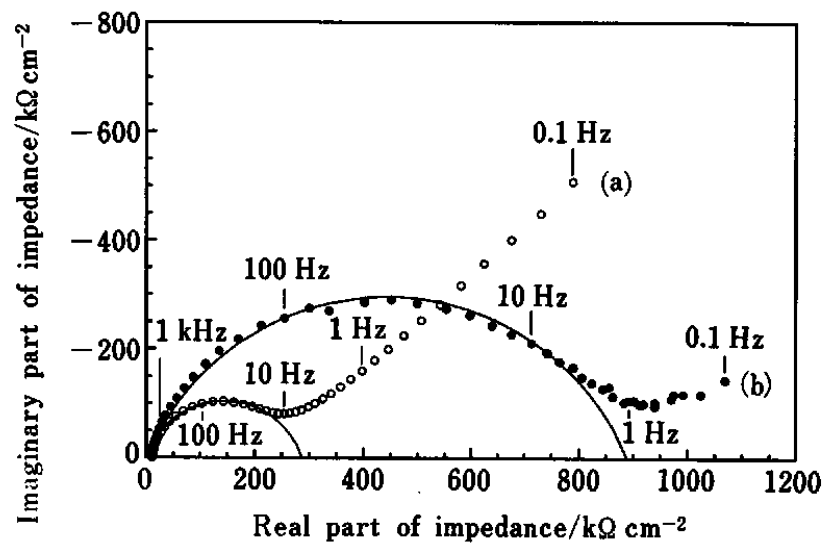

Fig. 5 Complex impedance plots for (a) bare gold electrode and (b) 1-modified gold electrode having $\theta$ value of 0.26 . Electrolyte solution: aqueous $0.1 \mathrm{M} \mathrm{KCl}$ containing $5 \mathrm{mM}$ each of $\mathrm{K}_{4}\left[\mathrm{Fe}(\mathrm{CN})_{6}\right]$ and $\mathrm{K}_{3}\left[\mathrm{Fe}(\mathrm{CN})_{6}\right]$; temperature, $25^{\circ} \mathrm{C}$. Measurements were made at $50 \mathrm{mV}(\mathrm{rms})$ around $E^{\prime}$ of the $\left[\mathrm{Fe}(\mathrm{CN})_{6}\right]^{3-14-}$ couple (typically, $+209-+217 \mathrm{mV}$ ). Solid lines show theoretical calculation according to Eq. (1) based on curve fitting to experimental data. 
equivalent circuit is reduced to a parallel combination of $C_{\mathrm{dl}}$ and $R_{\mathrm{CT}}$ (plus serial $R_{\mathrm{soln}}$ ). In a low frequency region on the other hand, the impedance is dominated by a diffusion of redox species and the equivalent circuit reduces simply to a serial combination of $R_{\text {soln }}$ and $Z_{\mathrm{W}}$, which now gives a $45^{\circ}$ straight line in the diagram. The whole impedance diagram therefore gives a semi-circle fused with a $45^{\circ}$ "diffusion" line. In Fig. 5, the bare gold electrode exactly shows these features. This means that Randles' equivalent circuit is properly applicable to this electrochemical system.

In case of the modified electrode, only a kinetic semicircle was observed, and a $45^{\circ}$ diffusion line at low frequencies did not show up clearly in the frequency range studied. The electrical parameters which determine the pattern of impedance diagram are $R_{\mathrm{CT}}$ and its relative magnitude to that of Warburg impedance. In case of the electrochemical system having a large $R_{\mathrm{CT}}$ (i.e., a charge transfer process is kinetically rather sluggish), the diagram shows a $45^{\circ}$ diffusion region only partially, because mass transfer is not a significant factor in most of the frequency range. As already noted, surface monolayer of 1 significantly blocks the electron transfer process at the electrode-solution interface. This should lead to a large $R_{\mathrm{CT}}$ value of the system, which in turn means an enlarged semicircle in the impedance diagram, leading to the lack of a clear $45^{\circ}$ linear portion in the plots. On the other hand, contrary to our first expectations, the monolayer of 1 did not totally block the redox reaction by the solution species. This may be due to the low $\theta$ value of the electrode. However, it should be emphasized that the impedance diagram itself is clear and reproducible enough to evaluate the a.c. parameters by adopting the Randles' equivalent circuit.

First, $R_{\mathrm{CT}}$ values were obtained by non-linear curve fitting procedure from the experimental impedance data obtained in the frequency region above $8 \mathrm{~Hz}$. In this frequency region, the impedance plots can be safely regarded as forming a part of a semi-circle. The following equation was used in the curve fitting calculation:

$$
I_{\mathrm{m}}=\left(-\left(R_{\mathrm{e}}-l\right)^{2}+m^{2}\right)^{1 / 2}+n
$$

where $R_{\mathrm{e}}$ and $I_{\mathrm{m}}$ stands for the real and imaginary parts of the impedance, respectively. The parameters $l$ and $n$ stand for the coordinate of the center of the circle, and $m$ stands for the radius of the circle. The $R_{\mathrm{CT}}$ values estimated were in the range $0.3-0.5 \mathrm{M} \Omega \mathrm{cm}^{-2}$ and $0.6-$ $1.8 \mathrm{M} \Omega \mathrm{cm}^{-2}$ for bare and 1-modified electrodes, respectively, for several independent electrode preparations. The electrical capacitance $C_{\text {exp }}$ can be evaluated from the frequency $f_{\max }$, which represents the upper maximum point of the semi-circle in the complex impedance plots, according to the relationship ${ }^{18}$

$$
\begin{aligned}
\omega_{\max } & =1 /\left(R_{\mathrm{CT}} C_{\exp }\right) \\
& =2 \pi f_{\max } .
\end{aligned}
$$

It seems reasonable to make a simple approximation that $C_{\text {exp }}$ is a parallel combination of the double-layer capacitance $C_{\mathrm{d} 1}$ at the unmodified, bare gold surface and the modified-layer capacitance $C_{\mathrm{m} .}{ }^{16}$ This gives the expression

$$
C_{\mathrm{m}}=\left[C_{\text {exp }}-C_{\mathrm{dl}}(1-\theta)\right] / \theta .
$$

The dielectric constant $\varepsilon$ of the modified layer (monolayer of 1) can then be calculated by assuming the monolayer thickness.

Unfortunately, the $C_{\mathrm{dl}}$ value of the bare gold electrode was found to be sensitive to such experimental uncertainties as the mean surface area (roughness of the electrode surface) and adsorbed impurities. Thus, we only obtained rather scattered values for $C_{\mathrm{dl}}$ ranging from 17 to $22 \mu \mathrm{F} \mathrm{cm}^{-2}$. For the modified electrode having $\theta$ value of 0.31 ; the $C_{\exp }$ value obtained was $17 \mu \mathrm{F} \mathrm{cm}^{-2}$. By assuming the thickness of $3.3 \mathrm{~nm}$ for the monolayer, which was estimated by CPK model building, the effective dielectric constant of the monolayer ranging from 22 to $57 \mu \mathrm{F} \mathrm{cm}^{-2}$ was calculated. The dielectric constant of simple alkanethiols aligned in the monolayer on $\mathrm{Au}$ surface is reported to be in the range $2-3 \mu \mathrm{F}$ $\mathrm{cm}^{-2}$; at first glance, the data obtained above in the present study look unusual. However, the dielectric constant of liquid nitrobenzene is $34.6 \mu \mathrm{F} \mathrm{cm}^{-2}$. In addition, as noted in the previous section, the monolayer of 1 was found to possess "liquid-like" nature on gold surface. If we consider these points, the relatively large monolayer dielectric constant obtained above might not be so unusual.

Impedance measurements were also made in the absence of $\left[\mathrm{Fe}(\mathrm{CN})_{6}\right]^{3-/ 4-}$ redox-couple in solution phase. Under such experimental conditions, the opencircuit potential can not be defined and can change. However, experimentally, the electrode potential measured under nitrogen did not show much change in the course of ordinary impedance measurement, staying typically between +0.45 and $+0.49 \mathrm{~V}(v s . \mathrm{Ag} / \mathrm{AgCl})$. A typical example of impedance diagram obtained under such conditions is shown in Fig. 6.

The profile at the $65 \mathrm{kHz}-6.3 \mathrm{~Hz}$ region is similar to that measured in the presence of redox couple. The profile in this frequency region was quite reproducible at measurements with independent electrode preparations. However, at $5 \mathrm{~Hz}$ and below, the data points began to scatter and the reproducibility of the measurement was lost. This seems inevitable since the system lacks redox species that are to carry out a net electron transfer reaction between the electrode and the solution phase. However, by using the data in the region above $6.3 \mathrm{~Hz}$ and by assuming the equivalent circuit similar to that adopted in Fig. 5, $R_{\mathrm{CT}}$ values ranging from 20 to $30 \mathrm{M} \Omega \mathrm{cm}^{-2}$ were calculated by a curve fitting procedure (Eq. (1)); these were one order larger than the $R_{\mathrm{CT}}$ values obtained in the presence of $\left[\mathrm{Fe}(\mathrm{CN})_{6}\right]^{3-/ 4-}$ couple. This $R_{\mathrm{Cr}}$ value also led to the $C_{\exp }$ values $\left(0.5 \mu \mathrm{F} \mathrm{cm}^{-2}\right)$ which were in fair agreement with those values $(0.2-$ 

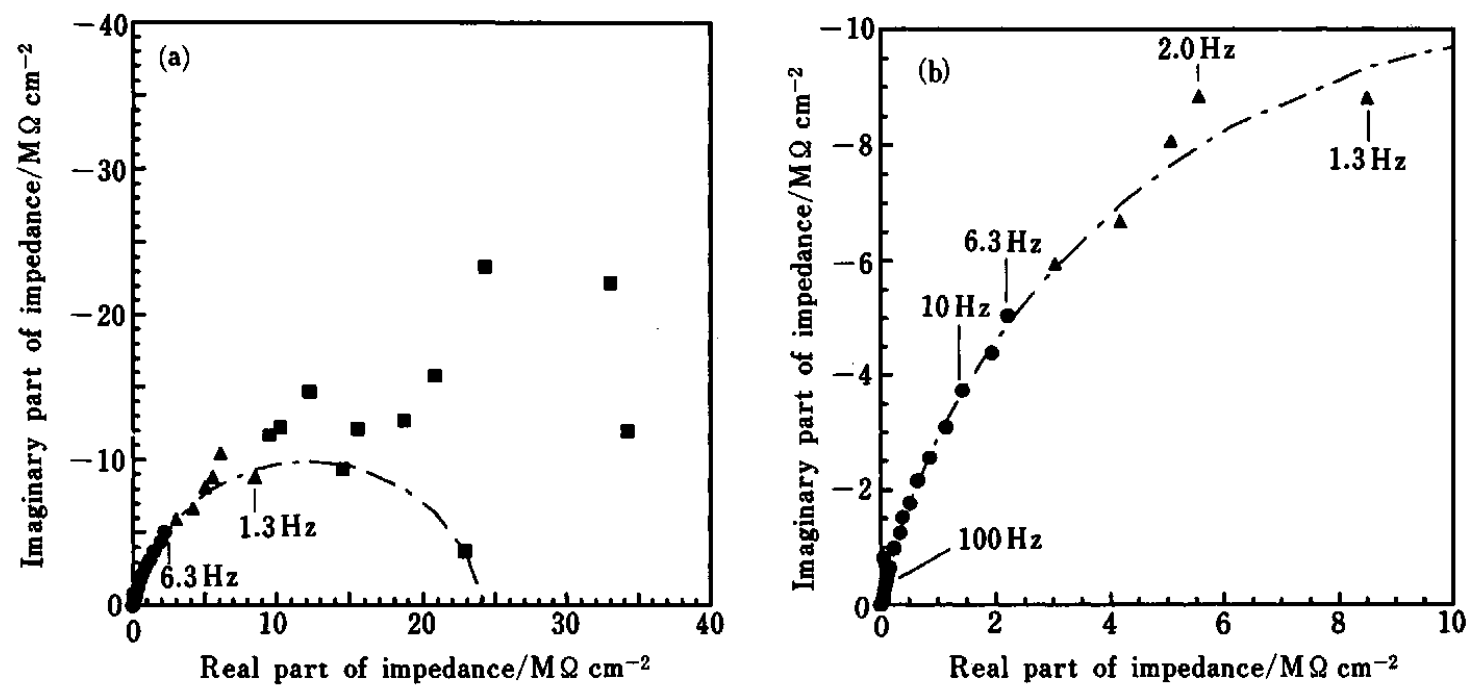

Fig. 6 Complex impedance plots for 1-modified gold electrode in the absence of $\left[\mathrm{Fe}(\mathrm{CN})_{6}\right]^{3-14-}$ redox couple. (a) Impedance diagram in the whole frequency region studied. $0,65 \mathrm{kHz}-$ $6.3 \mathrm{~Hz} ; \Delta, 5.0 \mathrm{~Hz}-1.3 \mathrm{~Hz} ; \mathbf{n}, 1.3 \mathrm{~Hz}-0.1 \mathrm{~Hz}$. (b) Impedance diagram in high-frequency region (enlarged). $\quad, 65 \mathrm{kHz}-6.3 \mathrm{~Hz} ; \Delta, 5.0 \mathrm{~Hz}-1.3 \mathrm{~Hz}$. Electrolyte solution: aqueous $0.5 \mathrm{M} \mathrm{KCl}$ containing $0.05 \mathrm{M}$ each of boric acid- $\mathrm{NaOH}$ buffer $\left(\mathrm{pH} 8.0\right.$ ); temperature, $25^{\circ} \mathrm{C}$. Broken lines show theoretical calculation based on curve fitting to experimental data at $65 \mathrm{kHz}-6.3 \mathrm{~Hz}$.

$0.6 \mu \mathrm{F} \mathrm{cm}^{-2}$ ) obtained in the presence of redox couple.

From a strict electrochemical viewpoint, measurements in the absence of redox couple are not adequate to study the precise nature of a chemically modified gold electrode. However, as shown in the observations above, impedance behavior of the modified electrode does not suffer from modification in the absence of redox couple if the measurements are limited in the high frequency region $(\geq 6.3 \mathrm{~Hz})$. Therefore, we decided to employ a redox couple-free system to explore the possibility of impedimetric immunosensing. The absence of redox couple is also expected, at the first approximation, to diminish chemical complexity of the system when the adsorption of a protein (anti-DNP antibody) is to be studied on the electrode surface.

\section{Application to impedimetric immunosensor}

We have described above the preparation and the characterization of 1-modified gold electrode. In the same way as in the previous section, FRA study was made for the 1-modified gold electrode in the presence of antibodies. The impedance diagram obtained was fundamentally similar to that in Fig. 6 (which was obtained without antibodies), but the plots shifted slightly upward. Because of the same difficulty as in Fig. 6, the intrinsic value of electrical capacitance of the modified layer itself could not be determined precisely. Further, an additional difficulty arose since the immunoreaction on the electrode surface influenced not only $C_{\mathrm{m}}$ but also $R_{\mathrm{CT}}$ values. A detailed discussion is beyond the scope of the present study. These circumstances led us to discuss the sensory application conveniently by using a combined electrical impedance which includes both $C_{\mathrm{m}}$ and $R_{\mathrm{cr}}$.
The electrical impedance of the 1-modified electrode was measured at $100 \mathrm{~Hz}$ while changing the concentration of antibody in the solution. The impedance was first measured in the absence of antibody, and the change or increment on the succeeding addition of the antibody was recorded. The results are summarized in Fig. 7. In the case of monoclonal anti-DNP antibody, the electrical impedance increased as the concentration of the antibody increased, while such concentration dependence was not observed in case of the addition of monoclonal anti-IgE antibody. These observations strongly suggest that the experimentally obtained antibody response arises from the binding of the specific antibody protein to the corresponding antigen portion available on the modified electrode surface. The impedance response is clear at the antibody concentration as low as $10 \mathrm{ng} \mathrm{cm}^{-3}$, but non-specific binding of anti-IgE antibody can not be negligible under similar concentration levels. The detection limit of $10 \mathrm{ng} \mathrm{cm}^{-3}$ is slightly less than that of the conventional immunoassays based on potentiometric ionophore-modulation ${ }^{6}$ and a piezoelectric ${ }^{7}$ principle.

Since the antibody molecules are electrically insulating, a piling-up of such molecules on the modified electrode surface is reasonably understood to decrease the electrochemical conductivity across the electrode/ modified layer/solution interface. Unfortunately, it is impossible at present to experimentally evaluate the effect of such antibody binding on the $C_{\mathrm{dl}}$ value. However, we can expect that the binding of nonpolar, organic molecules on an electrode surface reduces the $C_{\mathrm{di}}$ value. ${ }^{19}$

Although we have demonstrated positively the possibility of impedimetric immunosensing by using a 


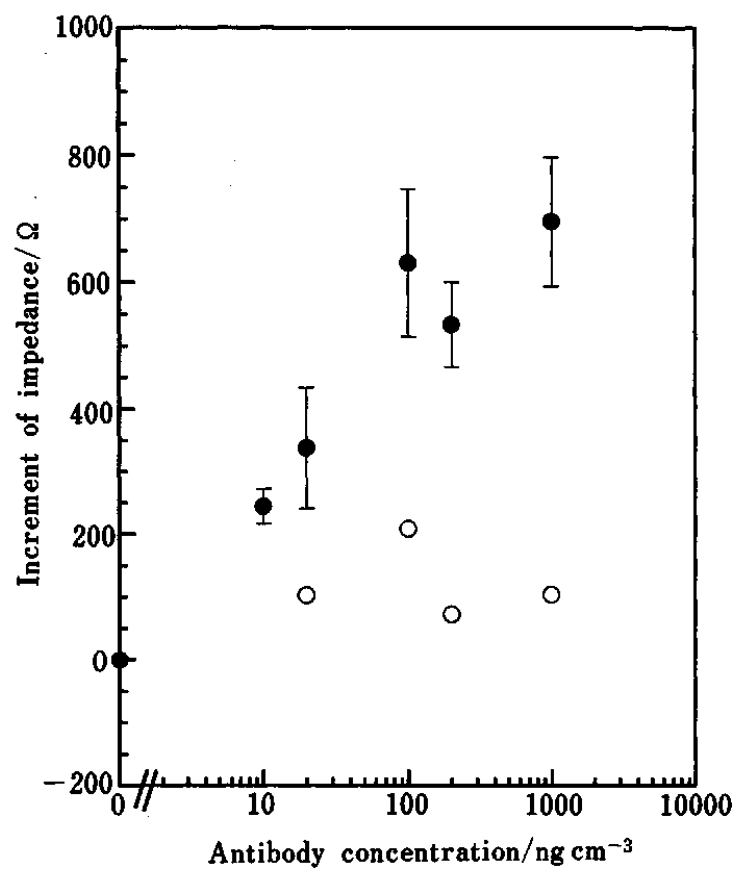

Fig. 7 Electrical impedance response of modified electrode to (O) anti-DNP antibody and (O) anti-IgE antibody. Frequency response analyses were made at $50 \mathrm{mV}$ (rms) around the natural potential (under nitrogen typically, $+450-+490 \mathrm{mV}$ ), and the change in electrical impedance at $100 \mathrm{~Hz}$ was plotted against the antibody concentration. Bars in the figure show variations in the response when the measurements were made with the two separate preparations of the electrode. Electrolyte solution: aqueous $0.5 \mathrm{M} \mathrm{KCl}$ containing $0.05 \mathrm{M}$ each of boric acid- $\mathrm{NaOH}$ buffer ( $\mathrm{pH} 8.0$ ); temperature, $25^{\circ} \mathrm{C}$.

modified gold electrode, the results presented here need to be supplemented with further investigations. One of the major problems is the variation of impedance according to the preparations of the modified electrode. For example, one preparation of 1-modified electrode showed an impedance value of $7.5 \mathrm{k} \Omega$ at $100 \mathrm{~Hz}$ in the absence of antibody. The electrode was repolished and remodified with 1 according to the standard procedure. The electrode thus obtained now showed the impedance value of $11 \mathrm{k} \Omega$. This variation clearly indicates a poor reproducibility of the roughness and the chemical reactivity of the polished gold surface. However, it is important that the response to the antibody concentration remained within a reasonably narrow variation range, as shown in Fig. 7 despite of this large absolute impedance difference in the independent preparations of the 1-modified electrodes.

The second problem is the possible interference caused by the adsorption of other proteins such as albumin, since the formed layer of proteins due to nonspecific adsorption other than immunochemical binding should also change the electrochemical impedance of the modified electrode. Therefore, influence of co-existing serum proteins on impedimetric response should be investigated before practical application of the electrode to blood samples. The development of biocompatible material on which protein adsorptions are prevented ${ }^{20}$ would hopefully give hints to overcome this problem.

We are grateful to Mr. H. Horiuchi, Kyushu University, for preparation of glassware items and electrodes. We also thank the Advanced Research Center of Kyushu University for access to a frequency response analyzer. This work was partially supported by a Grant-in-Aid for Scientific Research from the Ministry of Education, Science and Culture of Japan.

\section{References}

1. R. C. Murray, A. G. Ewing and R. A. Durst, Anal. Chem., 59, 379A (1987).

2. See for a recent summary: P. E. Laibinis, G. M. Whitesides, D. L. Allara, Y. Tao, A. N. Parikh and R. G. Nuzzo, J. Am. Chem. Soc., 113, 7125 (1991) and references cited therein.

3. C. E. D. Chidsey, C. R. Bertozzi, T. M. Putvinski and A. M. Mujsce, J. Am. Chem. Soc., 112, 4301 (1990).

4. A. E. G. Cass (ed.), "Biosensors-A Practical Approach", IRL Press, Oxford, 1990.

5. W. R. Heineman and H. B. Halsall, Anal. Chem., 57, 1321A (1985).

6. M. Y. Keating and G. A. Rechnitz, Anal. Chem., 56, 801 (1984).

7. H. Muramatsu, J. M. Dicks, E. Tamiya and I. Karube, Anal. Chem., 59, 2760 (1987).

8. P. Bataillard, F. Gardies, N. J. Renault, C. Martelet, B. Colin and B. Mandrand, Anal. Chem., 60, 2374 (1988).

9. T. Okuyama and K. Satake, J. Biochem., 47, 454 (1960).

10. V. Boekelheide, E. J. Corey, W. G. Dauben, W. D. Emmons, B. C. McKusick, W. E. Parham, J. D. Roberts and P. Yates (ed)., "Organic Synthesis Collective Volume", Vol. 4, p. 401, Wiley, New York, 1963.

11. R. B. Wagner and H. D. Zook, "Synthetic Organic Chemistry", p. 797, Wiley, New York, 1965.

12. J. R. Macdonald, "Impedance Spectroscopy. Emphasizing Solid Materials and Systems", Wiley Interscience, New York, 1987.

13. N. E. Herman and G. W. Siskind, Biochemistry, 3, 996 (1964).

14. R. G. Snyder, H. L. Strauss and C. A. Elliger, J. Phys. Chem., 86, 5145 (1982).

15. M. D. Porter, T. B. Bright, D. L. Allara and C. E. D. Chidsey, J. Am. Chem. Soc., 107, 3559 (1987).

16. E. Savatani, I. Rubinstein, R. Maoz and J. Sagiv, J. Electroanal. Chem. Interfacial Electrochem., 219, 365 (1987).

17. M. Sluyters and J. H. Sluyters, "Electroanalytical Chemistry-A Series of Advances", ed. A. J. Bard, Vol. 6, p. 1, Marcel Dekker, New York, 1970.

18. A. J. Bard and L. R. Faulkner, "Electrochemical Methods. Fundamentals and Applications", Wiley, New York, 1980.

19. B. B. Damaskin, O. A. Petrii and V. V. Batrakov, "Adsorption of Organic Compounds on Electrodes", Plenum Press, New York, 1971.

20. K. Ishihara, S. Ohta, T. Yoshikawa and N. Nakabayashi, J. Polym. Sci., Part A: Polym. Chem., 30, 929 (1992).

(Received October 20, 1992) (Accepted January 11, 1993) 